\title{
Faces evoke spatially differentiated patterns of BOLD activation and deactivation
}

\author{
Kevin A. Pelphrey, ${ }^{1,3}$ Peter B. Mack,' Allen Song,' Güven Güzeldere ${ }^{1,4}$ and Gregory McCarthy',2,CA \\ 'Duke-UNC Brain Imaging and Analysis Center, Duke University Medical Center, 163 Bell Building, Box 3918, Durham, NC 277I0; ${ }^{4}$ Department of Philoso- \\ phy, Duke University; ${ }^{2}$ Department of Veterans Affairs Medical Center Durham, NC; ${ }^{3}$ Neurodevelopmental Disorders Research Center, University of \\ North Carolina at Chapel Hill School of Medicine, Chapel Hill, NC, USA \\ ${ }^{\mathrm{CA}}$ Corresponding Author and Address
}

Received I November 2002; accepted II February 2003

DOI: 10.1097/0I.wnr.0000074345.81633.ad

\begin{abstract}
Using fMRI techniques sensitive to blood oxygen-level dependent (BOLD) contrast, we measured brain activity in participants $(n=8)$ as they viewed images of faces presented periodically within a continuously changing montage of common objects. Consistent with prior studies, we identified regions of ventral extrastriate cortex, primarily in the fusiform and inferior temporal gyri and nearby cortex, that were activated by faces as measured by an increase in BOLD signal. In addition, we made the novel observation
\end{abstract}

that faces deactivated other areas of ventral extrastriate cortex, primarily in the lingual and parahippocampal gyri and medial to activations. These deactivated regions, identified by a decrease in BOLD signal, may reflect populations of neurons that decrease their activity when faces appear, possibly as a consequence of category-specific inhibition. NeuroReport 14:000-000 (c) 2003 Lippincott Williams \& Wilkins.

Key words: BOLD deactivations; Face processing; fMRI; Gradient-recalled inverse spiral; Object processing

\section{INTRODUCTION}

Field potential recordings from subdural electrodes in humans have revealed that focal regions of ventral extrastriate cortex, principally within the lateral fusiform gyrus, respond selectively to faces but not to scrambled faces or exemplars from other categories such as butterflies or cars [1-5]. The initial face-specific response is evident as a cortical surface-negative potential with a peak latency of 180-200 ms (N200). At some other ventral sites that do not respond to faces, similar N200s are evoked selectively by strings of letters [5]. Results fMRI studies have also demonstrated selective activation evoked by faces and letterstrings in human occipitotemporal cortex [6-9]. For example, McCarthy et al. [6] measured changes in blood oxygen-level dependent (BOLD) contrast evoked by faces or flowers presented periodically in a continuously changing montage of everyday objects. Faces evoked activation within the fusiform gyrus, primarily within the right hemisphere, while flowers evoked no such activation, indicating that faces activated regions of the fusiform beyond those activated by common objects. However, when presented in a similar montage of non-object patterns with the same spatial frequencies of the common objects, both faces and flowers activated extensive bilateral regions of the fusiform gyri, indicating that some regions of ventral extrastriate cortex respond non-selectively objects and faces.
Part of the selectivity exhibited in extrastriate cortex may result from inhibitory interactions; for example, single unit studies in monkeys have shown that some neurons activated by one category of stimuli are inhibited by nonpreferred stimuli [10-12]. Allison et al. [13] recently presented evidence for such category-selective inhibitory interactions in humans. They identified word- and facespecific sites via subdural recordings made from the fusiform gyri and adjacent cortex. Faces evoked a surfacepositive potential (P200) at approximately one-half of wordspecific N200 sites. Conversely, words evoked P200 responses at about one-half of face-specific N200 sites. Allison et al. [13] argued that the P200 represents inhibition of category-specific neurons, and provided a model of synaptic connectivity between neurons selectively activated by faces and letterstrings to account for their result. While plausible, this model is unproven and converging evidence from other sources will be necessary to establish whether the phenomenon they describe relates to category-specific inhibition.

Task-related decreases in BOLD contrast (deactivations) have been reported in fMRI studies [14-17] and have been correlated with decreased blood oxygenation and neural suppression [17]. Functional neuroimaging studies could therefore provide the desired converging evidence by determining whether a stimulus category, such as faces, evokes spatially discrete BOLD contrast activations and 
deactivations. Unfortunately, blocked designs do not readily allow one to disambiguate BOLD contrast activation and deactivation. For example, in a blocked design in which faces alternate with letterstrings, it is ambiguous whether a voxel is deactivated by the presentation of faces, or whether that voxel was activated by letterstrings in the preceding block and is now returning to baseline during the face block (see Fig. 5 of 8 ).

To further investigate category-specific inhibitory mechanisms in human ventral extrastriate cortex, we measured changes in BOLD contrast as participants viewed faces presented periodically within a continuously changing montage of common objects. Because objects appear throughout the periods in which faces appear, any voxels activated by objects should remain active when faces appear. Thus, face-evoked BOLD contrast deactivations could be differentiated from activations evoked by common objects. We used frequency domain analysis to identify areas that were activated and deactivated by the appearance of faces among continuously present and changing common objects. Regions of inferior extrastriate cortex activated by faces occurred primarily within the fusiform and inferior temporal gyri. Importantly, we also identified regions of ventral occipitotemporal cortex that were deactivated by faces. Deactivations were most often located in the lingual and parahippocampal gyri, medial to face activations.

\section{MATERIALS AND METHODS}

Participants: Eight right-handed healthy volunteers (1933 years old; six female, two male) provided written consent to participate in this study. The Duke University Institutional Review Board approved this project.

Stimuli and experimental design: Two categories of grayscale images were shown to participants at XGA resolution using LCD display goggles. The categories were male and female faces (Fig. 1a) and images of individual common objects, fruits, and vegetables (Fig. 1b). Images from the categories were equated for intensity and contrast. Each $200 \mathrm{~s}$ run consisted of $\sim 1100$ stimuli, each presented for $500-1000 \mathrm{~ms}$ at randomly selected locations, resulting in a continuously changing montage in which an average of 3.85 stimuli were visible at any moment.

Two conditions were presented in a modified blocked design. The first condition (objects only) contained only objects (Fig. 1a). In the second condition (objects and faces), faces appeared among the objects (Fig. 1b). The average number of images displayed was equated for the two experimental conditions, which were interleaved in $17.46 \mathrm{~s}$ cycles, consisting of one objects only block (8.73 s) and one objects and faces block (8.73s) per cycle (Fig. 1c). Eleven cycles occurred in each run, and all participants completed 12 imaging runs. Six seconds of the objects only block appeared at the beginning of each run. Odd runs began with objects and faces and even runs began with the objects only condition. Two participants viewed alternative stimuli. Objects only blocks remained identical while objects and faces blocks were modified so that equal numbers of objects were presented in both objects only and objects and faces blocks, resulting in more images shown during objects and faces blocks.

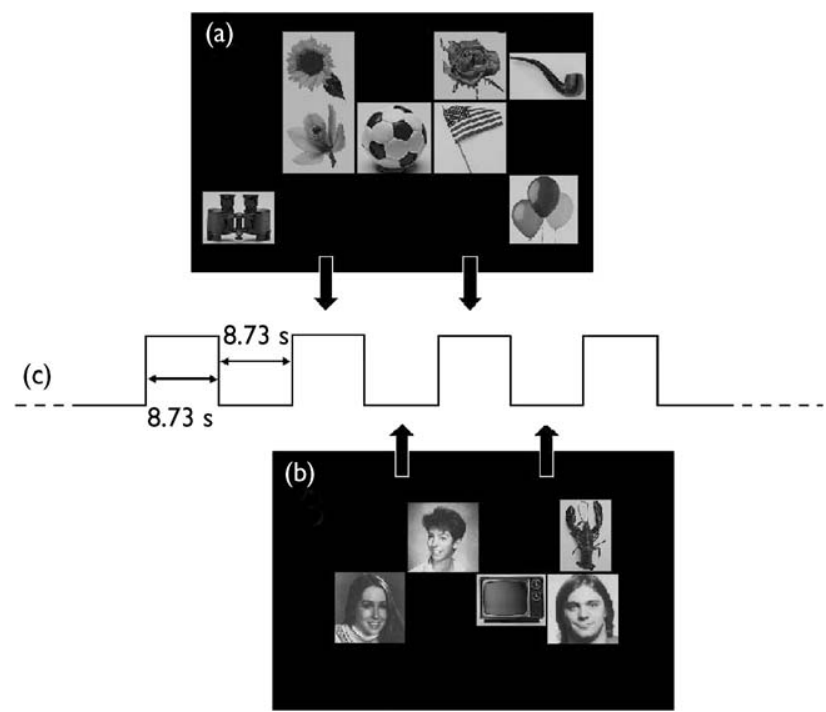

Fig. I. (a) The objects only condition. (b) The objects and faces condition. C: Objects only periods lasted $8.73 \mathrm{~s}$ and alternated with $8.73 \mathrm{~s}$ objects and faces periods.

Imaging parameters: Scanning was performed on a General Electric $4 \mathrm{~T}$ LX NVi MRI scanner system equipped with $41 \mathrm{mT} / \mathrm{m}$ gradients, and using a quadrature birdcage radio frequency $(\mathrm{RF})$ head coil for transmit and receive (General Electric, Milwaukee, Wisconsin, USA). Sagittal localizer images were acquired and used to define a series of 60 axial images acquired using 3D SPGR pulse sequence (TR, $450 \mathrm{~ms}$; TE, $20 \mathrm{~ms}$; FOV, $24 \mathrm{~cm}$; image matrix, 256 ${ }^{2}$; voxel size, $0.9375 \times 0.9375 \times 3.8 \mathrm{~mm})$. We selected 20 contiguous $3.8 \mathrm{~mm}$ axial slices for prescription of BOLD contrast images. A semi-automated high-order shimming program preceded the acquisition of functional images to ensure global field homogeneity. Functional images were acquired inferior to superior, with the first slice anchored $3.8 \mathrm{~mm}$ inferior to the ventral-most portion of the occipital lobe. Functional images were collected using a gradient-recalled inverse spiral imaging pulse sequence [18] sensitive to BOLD contrast (TR, $1000 \mathrm{~ms}$; TE, $30 \mathrm{~ms} ; \mathrm{FOV}, 24 \mathrm{~cm}$; image matrix, $64^{2} ; \alpha=62^{\circ}$; voxel size $3.8 \times 3.8 \times 3.75 \mathrm{~mm}$ ). Each imaging run consisted of 200 time points per image slice and began with four discarded RF excitations.

We initially identified the activation/deactivation phenomenon in a pilot study conducted at $1.5 \mathrm{~T}$. Here, the use of the inverse spiral imaging technique facilitated recovery of signal from ventral areas at $4 \mathrm{~T}$, as the high spatial frequency regions in k-space were sampled at shorter effective echo times that led to smaller intra-voxel dephasing effect. The shortened effective echo times, however, may result in moderate reduction of the BOLD sensitivity at the ventral brain regions, since a reasonably long TE is required to gain optimal BOLD contrast. Nevertheless, the benefit on the significant signal recovery overweighs the small loss of the BOLD sensitivity.

Data analysis: Each participant's data were analyzed using frequency domain analysis [12-14]. Runs were averaged by first block type, creating two average runs each consisting of 200 images. One was the average of all 

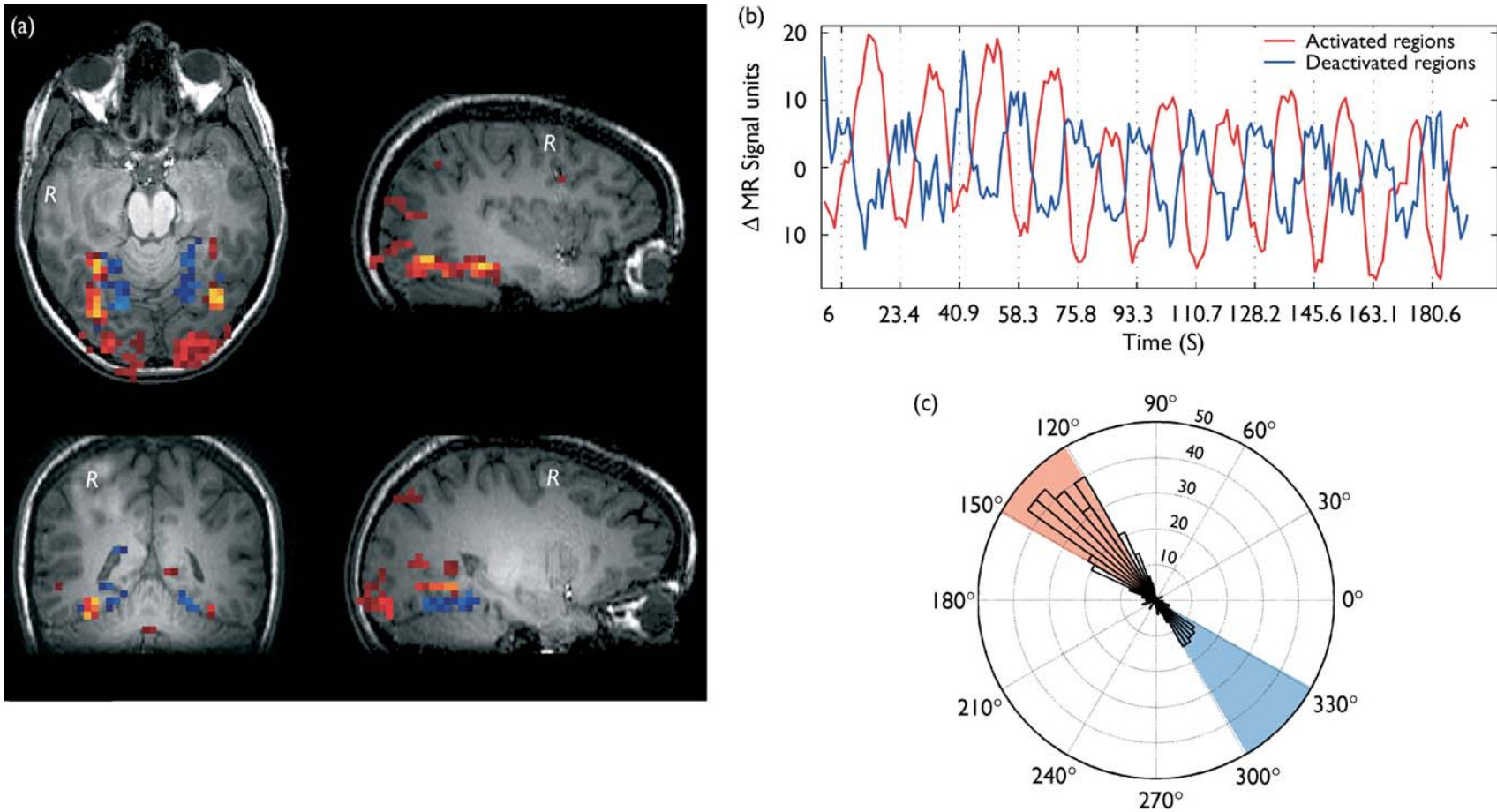

Fig. 2. (a) Power spectra data overlaid on anatomic images from a single participant. Shown are face-activated (red to yellow color map) and facedeactivated (dark to light blue color map) regions. Lighter color shading represents higher power. (b) Time courses of MR signal intensity for the faceactivated (red line) and face-deactivated (blue line) voxels in ventral extrastriate cortex. Vertical dashed lines depict the onset of each $8.73 \mathrm{~s}$ period containing faces. (c) Counts of face-related voxels by phase angle. Most face-related voxels were within ranges demarcating face-activated (136 $\pm 15^{\circ}$ : shaded in red) and face-deactivated $\left(316.6 \pm 15^{\circ}\right.$ : shaded in blue) voxels.

runs beginning with an objects only block. The other was the average of all runs beginning with an objects and faces block. Using Fourier transformation, frequency and phase spectral estimates for each voxel were computed for both time series. From the frequency spectrum for a given voxel, the percent variance at $\sim 0.057 \mathrm{~Hz}(1 / 17.46 \mathrm{~s})$ was calculated, and was averaged across the odd and even numbered runs. Voxels having at least $15 \%$ of their variance attributable to signal at $0.057 \mathrm{~Hz}$ were deemed face-related.

The phase angle at $0.057 \mathrm{~Hz}$ for all voxels in the even numbered runs was shifted $180^{\circ}$ to compensate for the shift in stimulus presentation, then the phase angles for the even and odd numbered runs were averaged. Accounting for the $6 \mathrm{~s}$ of objects at the beginning of each run and a $5 \mathrm{~s}$ hemodynamic response lag, a signal peaking at the middle of objects and faces blocks has a phase angle of $136.6^{\circ}$. All voxels with sufficient power at $0.057 \mathrm{~Hz}$ were deemed facerelated and those with a phase angle of $136.6 \pm 15^{\circ}$ were considered face-activated. Conversely, those voxels that were face-related but had a phase angle that differed by $180^{\circ}$, which is $316.6 \pm 15^{\circ}$, were considered face-deactivated. Face-activated voxels varied with the blocks having a peak $\sim 5 \mathrm{~s}$ after the middle of an objects and faces block while face-deactivated voxels varied with blocks having a trough $\sim 5 \mathrm{~s}$ after the middle of an objects and faces block.

\section{RESULTS AND DISCUSSION}

Figure 2a shows face-activated (red to yellow color map) and face-deactivated (dark to light blue color map) voxels from a single participant, overlaid on corresponding anatomical images. We focused on the distribution of faceactivated and face-deactivated regions in extrastriate cortex. We localized clusters of face-activated voxels to the fusiform and inferior temporal gyri and nearby cortex. Clusters of face-deactivated voxels were localized medial to the faceactivated voxels, primarily in the lingual and parahippocampal gyri. The pattern of responsivity displayed in Fig. 2a is representative of that observed in the other seven participants (Fig. 3). The coupling of activation and deactivation is illustrated in Fig. 2b. Peaks of the time course from face-activated voxels coincided with the midway points of the objects and faces stimulus blocks (after accounting for hemodynamic lag). Troughs of the time course from face-deactivated voxels coincided with the same midway points. Counts of face-activated and facedeactivated voxels are displayed by phase angle in Fig. 2c. As expected, most of the face-related voxels fell within the two $30^{\circ}$ ranges demarcating face-activated $\left(136.6 \pm 15^{\circ}\right)$ and face-deactivated $\left(316.6 \pm 15^{\circ}\right)$.

Examples of activation and deactivation from the eight individual participants are displayed in Fig. 3. Each numbered image pair (1-8) corresponds to a single individual. The extent and localization of face-activated and face-deactivated clusters varied. However, the pattern of interest, a region of face-activated voxels and a region of face-deactivated voxels, was commonly observed. Counts of face-related voxels in ventral extrastriate cortex were averaged across participants. Most of the face-related voxels had phase angles within the face-activated and facedeactivated ranges. On average, there were more face- 

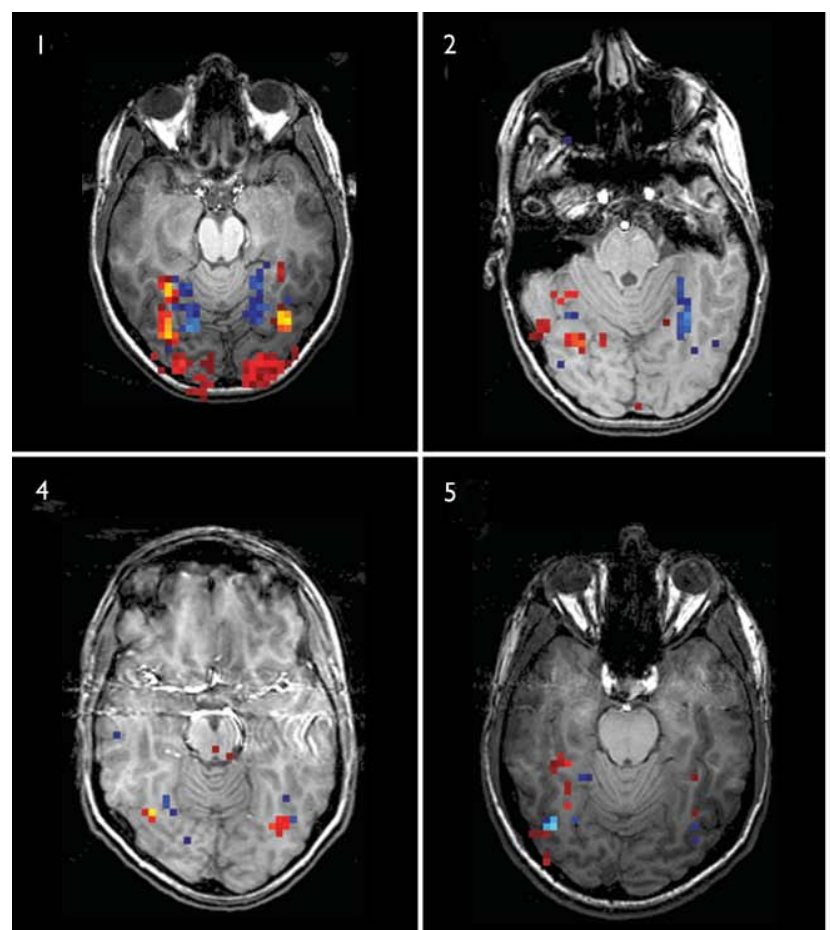
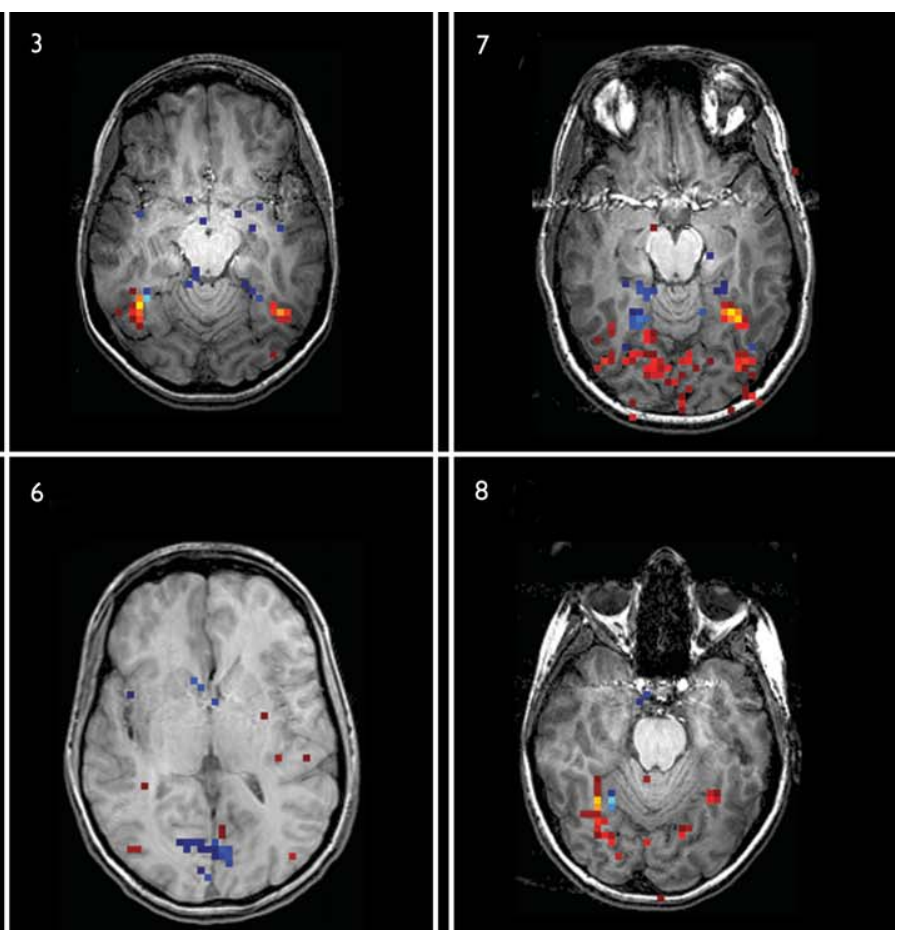

Fig. 3. Power spectra data overlaid on anatomical images from each of the eight participants (one axial and one coronal image per individual). Shown are face-activated (red to yellow color map) and face-deactivated (dark to light blue color map) regions. Lighter shading represents higher power. Images follow radiographic convention. Face-activated and face-deactivated regions were localized to ventral extrastriate cortex, with face-deactivated areas often medial to face-activated areas. Participants I-6 received the original stimulus protocol and participants $7-8$ viewed alternative stimuli.

activated $(79.63 \pm 27.22$, s.e. $)$ than face-deactivated $(33.63 \pm 8.26)$ voxels in ventral extrastriate cortex, with a ratio of $\sim 2.3: 1$.

Our results replicate previous findings [12-14]. Faces activated a region of ventral extrastriate cortex localized primarily to the fusiform and inferior temporal gyri. We also extended prior reports, revealing areas of ventral extrastriate cortex that deactivated to faces, localized to the lingual and parahippocampal gyri, medial to activations. These deactivations could reflect populations of neurons that decrease activity when faces are present, possibly because they are selectively involved in processing other non-face object categories. The decreases in BOLD signal observed in face-deactivated areas are consistent with the presence of a remote inhibitory signal originating from the activity of neurons within the face-activated region.

An alternative is that the deactivated regions simply reflect the reduction in the number of objects present during objects and faces blocks compared to objects only blocks. Comparison of the findings from participants receiving the original and alternative stimulus protocols allowed us to test this alternative. Six of the eight participants (1-6 of Fig. 3) viewed the original protocol in which the average number of images remained constant across object only and object and face blocks (i.e. fewer objects appeared during object and faces blocks because faces replaced objects). The remaining two participants (7-8 of Fig. 3) viewed alternative stimuli in which objects only blocks remained identical while objects and faces blocks were modified so that the same numbers of objects were presented as in the objects only blocks. We reasoned that one offering the alternative explanation would predict the absence of deactivation in participants viewing the alternative stimuli. Comparison of the images from participants that viewed the original stimulus protocol (1-6 of Fig. 3) to those that viewed the alternative stimuli (7-8 of Fig. 3) reveals that the activation/deactivation pattern of responsivity was observed equally in the two groups. The alternative explanation cannot readily accommodate this observation.

Our results could reflect preferential distribution of attention to the faces as they appeared in the changing montage of common objects. That is, faces might draw attention away from the common objects, thus causing areas in processing non-face objects to deactivate. It is possible that face-specific cells (for example) inhibit object-specific cells primarily when attention is directed to faces, or it may be an automatic process regardless of the focus of attention. Prior studies have demonstrated effects of this kind. Using positron emission tomography (PET) to measure changes in regional cerebral blood flow $(\mathrm{rCBF})$ during somatosensory and visual tasks, Kwashima et al. [15] observed task-related deactivations in sensory areas involved in processing information directed to the irrelevant or unattended sensory modality. Similarly, in an fMRI study by Laurienti et al. [16], auditory stimuli elicited activation in auditory cortex and deactivation in extrastriate visual cortex and vice versa. These two studies demonstrated cross-modal inhibition of activity in sensory cortices. The present study demonstrates possible cross-category inhibition within extrastriate visual cortex. 
Our results could arise from the so-called vascular-steal phenomenon. That is, when there is an increase in local blood flow within the brain, adjacent areas will show a decrease in flow simply to meet the needs of the activated tissue. Gusnard and Marcus [19] suggested that this phenomenon has little relevance for fMRI studies of cognitive processes because the changes in local blood flow associated with increased cognitive/perceptual activity are quite small (a few percent locally in the cortex) relative to the overall blood flow to the brain. Moreover, they argued, the hemodynamic reserve of the brain is very large, making vascular-steal unlikely in the case of small changes in blood flow related to changes in processing. Finally, as observed in the present study, decreases sometimes occur remote from activations, or in the absence of increases, making steal within a given vascular territory an unlikely explanation for the current findings.

Cross-category inhibition may be a general feature of the human ventral object-recognition system. It may serve to suppress activity of non-preferred cells, and thus would act as a higher level sharpening mechanism. Because common objects were always present in the semi-blocked design of the current study, we were not able to assay the response of ventral extrastriate regions to common objects. We expect that the regions of deactivation observed in the current study would respond with an increase in activity to exemplars of other non-face object categories.

Finally, whereas we favor an interpretation of our results that emphasizes possible category specificity for faces in ventral extrastriate cortex, it is possible that the present findings reflect the operation of a particular type of visual processing that is engaged more by faces than common objects. Consistent with this interpretation, Farah [20] proposed that object recognition relies upon two processing systems, one a configural or holistic processing system required for normal face recognition, and the other a feature-based system required to recognize words. Both systems are useful for recognizing exemplars from other object categories. Our results, while certainly not conclusive on this point, might reflect the operation of the configural processing system, rather than a face subsystem per se.

\section{CONCLUSION}

Consistent with prior reports [6-8], we identified regions of inferior extrastriate cortex that activated to faces presented in a complex scene containing objects. Face-activated areas were localized to the fusiform and inferior temporal gyri and adjacent cortex. In addition, our results revealed facedeactivated regions of occipitotemporal cortex, localized to the lingual and parahippocampal gyri, generally medial to activations. Our findings offer important implications for the ways in which we conceptualize face and object processing in ventral extrastriate cortex. The newly identified face-deactivated areas could contain populations of neurons that decrease activity when faces are present, possibly because they are selectively involved in processing other non-face object categories. Prior studies have demonstrated cross-modal inhibition of activity in sensory cortices $[15,16]$. The present fMRI study is the first to demonstrate possible cross-category inhibition within extrastriate visual cortex. The observed BOLD contrast deactivations suggest the presence of an inhibitory signal originating from the activity of neurons within the face-activated region.

\section{REFERENCES}

1. Puce A, Allison T and McCarthy G. Cerebr Cortex 9, 445-458 (1999).

2. Allison T, Ginter H, McCarthy G et al. J Neurophysiol 71, 821-825 (1994).

3. Allison T, Puce A, Spencer DD and McCarthy G. Cerebr Cortex 9, 415-430 (1999).

4. Nobre AC, Allison T and McCarthy G. Nature 372, 260-263 (1994).

5. McCarthy G, Puce A, Belger A and Allison T. Cerebr Cortex 9, 431-444 (1999).

6. McCarthy G, Puce A, Gore JC and Allison T. J Cogn Neurosci 9, 605-610 (1997).

7. Puce A, Allison T, Gore JC and McCarthy G. J Neurophysiol 74, 1192-1199 (1995).

8. Puce A, Allison T, Asgari M et al. J Neurosci 16, 5205-5215 (1996).

9. Kanwisher N, McDermott J and Chun MM. J Neurosci 17, 4302-4311 (1997).

10. Baylis GC, Rolls ET and Leonard CM. Brain Res 342, 91-102 (1985).

11. Perrett DI, Oram MW, Harries MH et al. Exp Brain Res 86, 159-173 (1991).

12. Tamura H and Tanaka K. Cerebr Cortex 11, 384-399 (2001).

13. Allison T, Puce A and McCarthy G. J Neurophysiol 88, 2864 (2002).

14. Huettel SA, Güzeldere G and McCarthy G. J Cogn Neurosci 13, 1006-1018 (2001).

15. Kawashima R, O'Sullivan BT and Roland PE. Proc Natl Acad Sci USA 92, 5969-5972 (1995).

16. Laurienti PJ, Burdette JH, Wallace MT et al. J Cogn Neurosci 14, 420-429 (2002).

17. Wenzel R, Wobst P, Heekeren HH et al. J Cerebr Blood Flow Metab 7, 1103 1110 (2000).

18. Glover GH and Law CS. Magn Reson Med 46, 515-522 (2001).

19. Gusnard DA and Raichle ME. Nature Rev Neurosci 2, 685-694 (2001).

20. Farah MJ. Specialization within visual object recognition, Clues from prosopagnosia and alexia. In: Farah MJ and Ratcliff G (eds). The Neuropsychology of High-level Vision. Hillsdale: Lawrence Erlbaum; 1994, pp. 133-146. 


\begin{tabular}{|c|}
\hline LIPPINCOTT \\
WILLIAMS AND WILKINS
\end{tabular}

IOURNAL NAME ARTICLE NO:
WNR

\section{QUERIES AND / OR REMARKS}

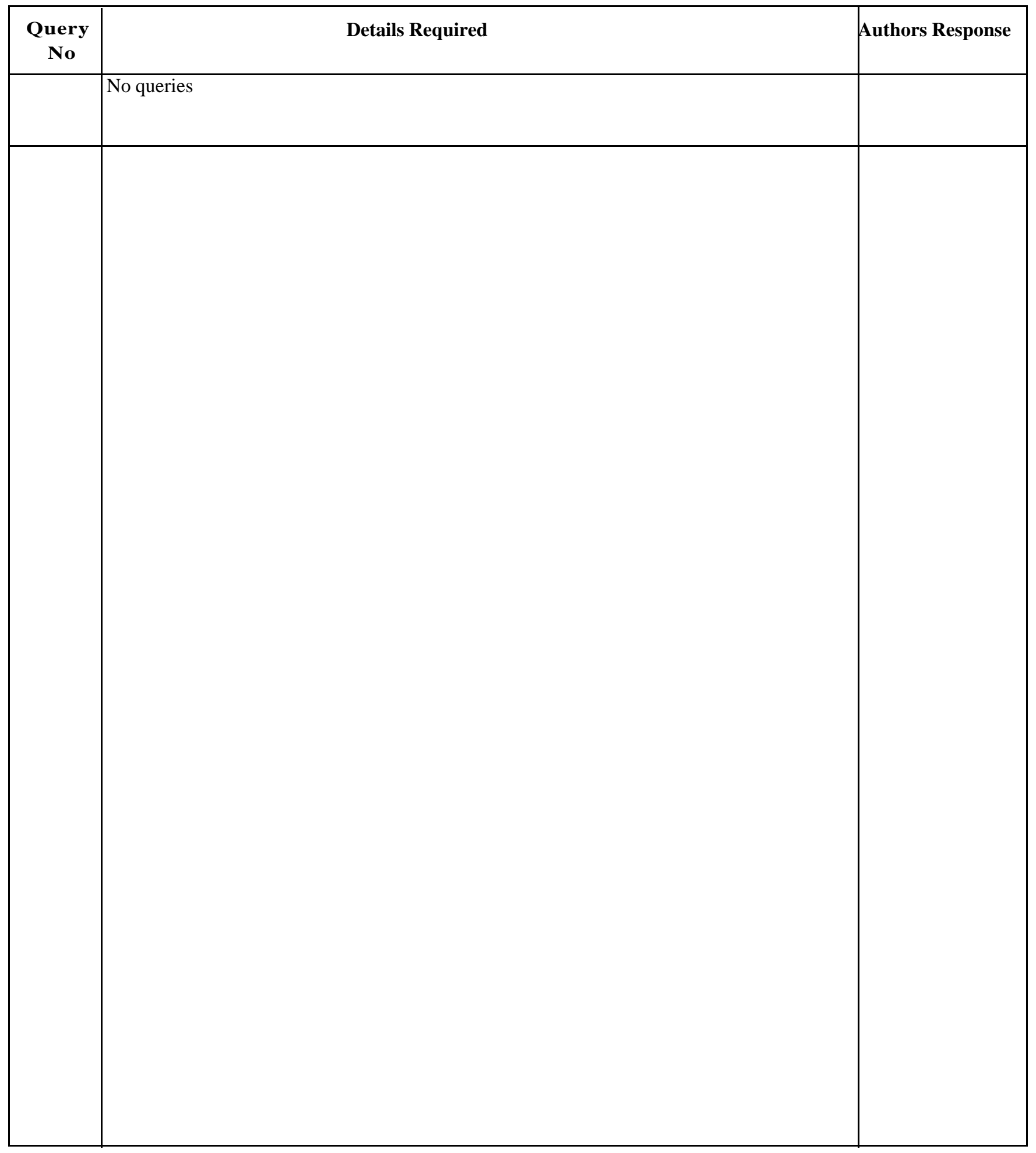

